\title{
Experimental and Theoretical Atomic-Resolved EELS Studies on Nitrogen Doped Single-Walled Carbon Nanotubes
}

\author{
Raul Arenal1,2, Katia March3, Chris P. Ewels4, Xavier Rocquefelte1, Mathieu Kociak3, Annick \\ Loiseau5, Odile Stéphan3 \\ 1. Laboratorio de Microscopias Avanzadas (LMA), Instituto de Nanociencia de Aragon (INA), \\ Universidad de Zaragoza, Calle Mariano Esquillor, 50018 Zaragoza, Spain
}

2. ARAID Fundation, Calle Mariano de Luna, 50018 Zaragoza, Spain

3. Laboratoire de Physique des Solides (LPS), CNRS UMR 8502, Université Paris Sud XI, Bâtiment 510, 91405 Orsay, France

4. Institut des Matériaux Jean Rouxel (IMN), CNRS UMR6502, Université de Nantes, 44322 Nantes,

France

5. Laboratoire d'Etude des Microstructures (LEM), UMR 104 CNRS-ONERA, 29 Avenue de la Division Leclerc, 92322 Châtillon, France

Having access to the chemical environment at the atomic level of a dopant in a nanostructure is crucial for the understanding of its properties. A very good example in this context is the case of notably nitrogen-doped carbon nanotubes $\left(\mathrm{CN}_{\mathrm{x}}-\mathrm{NT}\right)$. These nanomaterials have attracted much attention because of their interesting physical and chemical properties $[1,2]$. These properties are significantly affected by the atomic arrangement of the dopant atoms in such nanostructures [1-4]. Thus the knowledge of this information requires precision measurements, combining high spatial resolution and high spectroscopic sensitivity. In order to achieve these goals, we have developed atomically-resolved EELS allowing us to detect individual nitrogen dopants in single-walled carbon nanotubes. These results have been compared with first principles calculations.

The STEM-EELS-experiments were performed in a NION UltraSTEM 200, operated at $60 \mathrm{kV}$. The energy resolution was about $0.7 \mathrm{eV}$. In parallel, HRTEM imaging studies have been performed using an imaging-side aberration-corrected FEI Titan-Cube microscope working at $80 \mathrm{kV}$. Figure 1 displays a HAADF image of a single-walled (SW) CNx-NT where an EEL spectrum-image (39x45 recorded spectra $(1.95 \times 2.25 \mathrm{~nm})$, step size $0.05 \mathrm{~nm}$, probe size $\sim 0.11 \mathrm{~nm}$, acquisition time $100 \mathrm{~ms} / \mathrm{spectrum})$ has been recorded in the red marked area of the image. Three single EEL spectra, extracted from this spectrum-image (SI), in the marked positions/pixels of Fig. 1 (b) (spectra labelled (i), (ii) and (iii)), the 4 th spectrum is the sum of (i) and (ii). The carbon K edge is visible in the three spectra. In only two of the spectra of the whole dataset (1755 spectra), the nitrogen signal is also detectable. The nitrogen $1 \mathrm{~s}$ (N1s) ELNES, expanded in Figure 1 (c), show a strong peak at $\sim 401 \mathrm{eV}$, with very little signal at energies above this. Comparing the spectra to density functional theory (DFT) ELNES calculations of possible single nitrogen defects, there is excellent agreement with the spectrum for substitutional nitrogen (Fig. 1 (c)(iii) and atomic model, Fig. 1(d)) across the range of $\pi^{*}$ and $\sigma^{*}$ bands. We have also investigated other more complex configurations that we will present and discuss in this contribution [5]. In summary, these studies elucidate a crucial question concerning the nature of the nitrogen atomic configuration of CNx-NTs. In fact, this detailed knowledge of how nitrogen atoms are incorporated in the carbon lattice as well as precisely control of their incorporation are required for the use of these NTs for future technological applications.

\section{References}

[1] R. Arenal, X. Blase, A. Loiseau, Advances in Physics 59, 101 (2010). 
[2] P. Ayala, R. Arenal, A. Rubio, A. Loiseau, T. Pichler, Rev. Mod. Phys. 82, 1843 (2010).

[3] P. Ayala, R. Arenal, M. Rummeli, A. Rubio, T. Pichler, Carbon 48, 575 (2010).

[4] C.P. Ewels, M. Glerup, J. Nanosci. Nanotech. 5, 1345 (2005).

[5] R. Arenal, K. March, C.P. Ewels, X. Rocquefelte, M. Kociak, A. Loiseau, O. Stéphan, submitted.

[6] The research leading to these results has received funding from the European Union Seventh

Framework Program under Grant Agreement 312483 - ESTEEM2 (Integrated Infrastructure Initiative I3) and from the French CNRS (FR3507).

Figure 1. (a) HAADF image displaying atomic resolution (scale bar: $1 \mathrm{~nm}$ ). The red dotted rectangle marks out the scanned area during the acquisition of an EELS spectrum-image (SI). (b) Selection of EEL spectra extracted from the SI; blue, red and green spectra correspond to the pixels outlined in blue, red and green in the HAADF image acquired simultaneously with the SI and displayed in this figure. Each curve corresponds to a single spectrum from the SI, except the black, which is a sum of the blue and red spectra. (c) Simulated N1s ELNES (grey, (iii)) and nitrogen partial DOS calculations ((i) purple $=\mathrm{p}_{\mathrm{z}} \pi^{*}$-states, (ii) green $=\mathrm{p}_{\mathrm{x}-\mathrm{y}} \sigma^{*}$ states) for substitutional nitrogen, compared to the experimental spectrum (iv), same as spectra (i)+(ii) of Fig. 1 (b). The EELS simulations allow unambiguous assignment of the peak at $\sim 401 \mathrm{eV}$ in the N1s edge to a substitutional configuration shown in the DFT optimized structure of a substitutional nitrogen atom displayed in Fig. 1 (d). Carbon and nitrogen atoms are respectively in gray and blue.

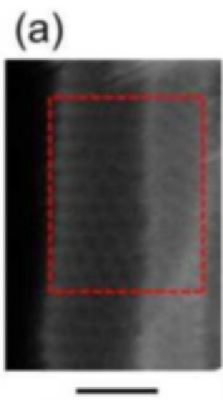

(d)

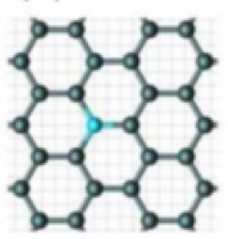

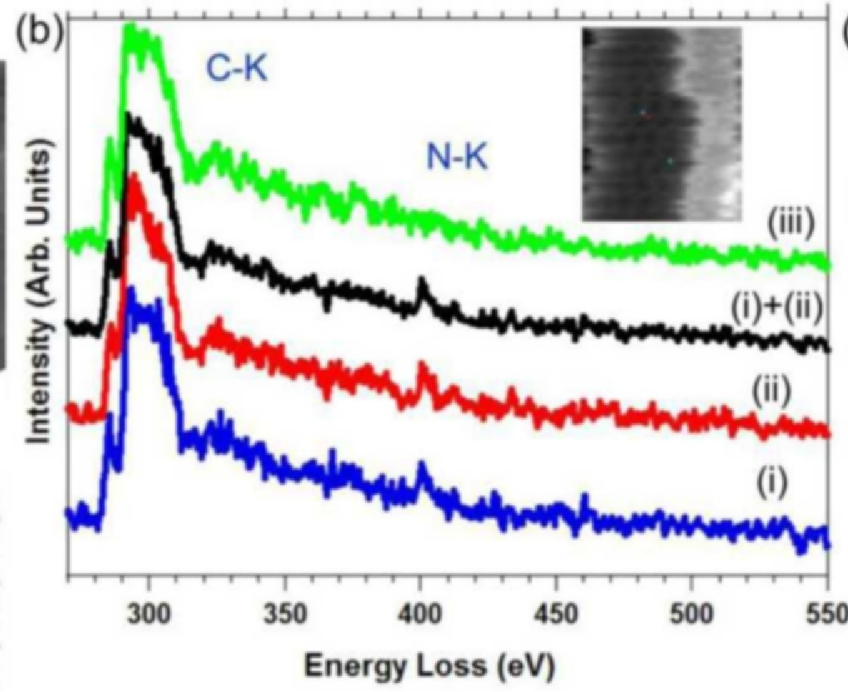

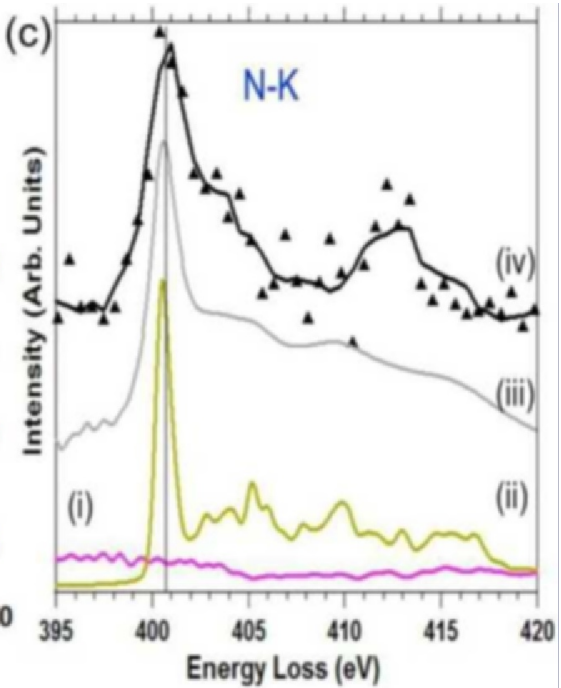

